\title{
REGULARITY AND $\sigma$-ADDITIVITY OF STATES ON QUANTUM LOGICS
}

\author{
MIRKO NAVARA
}

(Communicated by Palle E. T. Jorgensen)

\begin{abstract}
In 1977, Béaver and Cook [1] introduced the notion of regularity of states on quantum logics. They presented a generalization of Alexandroff theorem: each regular finitely additive state on a quantum logic is countably additive. Recently, Dvurečenskij, Neubrunn, and Pulmannová [2] observed an incorrectness in the original proof and doubted thus the validity of the result. We construct here a counterexample.
\end{abstract}

Let us briefly recall necessary notions (see [1] and [2]). (More details on quantum logics can be found in [4] and [5].)

By a (quantum) logic we mean an orthomodular poset $\mathscr{L}$. It is called $\sigma$ orthocomplete if each countable orthogonal sequence has a join in $\mathscr{L}$. A state on a quantum logic $\mathscr{L}$ is a mapping $\omega: \mathscr{L} \rightarrow[0,1]$ such that (i) $\omega(1)=1$, (ii) $\omega(a \vee b)=\omega(a)+\omega(b)$ whenever $a \perp b$. A state $\omega$ is called countably additive if $\omega\left(\bigvee_{n \in N} a_{n}\right)=\sum_{n \in N} \omega\left(a_{n}\right)$ whenever $\left\{a_{n}\right\}_{n \in N}$ is an orthogonal sequence in $\mathscr{L}$ having a join.

Suppose that $\mathscr{P}$ is a subset of $\mathscr{L}$. A state $\omega$ on $\mathscr{L}$ is called $\mathscr{P}$-regular if for each $\varepsilon>0$ and each $q \in \mathscr{L}$ there exists $p \in \mathscr{P}$ with $p \leq q$ and $\omega\left(q \wedge p^{\prime}\right)<\varepsilon$ (here $p^{\prime}$ means the complement of $p$ ). The set $\mathscr{P}$ is called finitely coverable if for each $p \in \mathscr{P}$ and each sequence $\left\{p_{n}\right\}_{n \in N} \subseteq \mathscr{P}$ such that $\bigvee_{n \in N} p_{n}^{\prime}$ exists and $p \leq \bigvee_{n \in N} p_{n}^{\prime}$ there exists a $k \in N$ satisfying $p \leq \bigvee_{n \leq k} p_{n}^{\prime}$.

The theorem of Béaver and Cook we want to consider was formulated as follows: Let $\mathscr{L}$ be a $\sigma$-orthocomplete quantum logic and let $\mathscr{P}$ be a finitely coverable subset of $\mathscr{L}$ containing the meet of any sequence in $\mathscr{P}$. Then each $\mathscr{P}$-regular state on $\mathscr{L}$ is countably additive.

In [2] the authors showed that the original proof of Béaver and Cook was applicable only for subadditive states. They also proved that the Béaver-Cook theorem was not valid for quantum logics which were not $\sigma$-orthocomplete. They left open the validity of the original result. As we shall show here, there is a counterexample to the Béaver-Cook theorem. The method for obtaining it is however quite different from that of [2].

Let us start with a finite (lattice) logic $\mathscr{S}$ admitting no states (see e.g. [3]). Let us take a trivial logic $\mathscr{T}=\left\{0_{\mathscr{T}}, 1_{\mathscr{V}}\right\}$. (We use indices following the

Received by the editors November 29, 1990.

1991 Mathematics Subject Classification. Primary 28A60, 81P20.

Key words and phrases. Quantum logic, regular states, countable additivity. 
symbols $0,1, \leq, \wedge, \vee$, etc. in order to distinguish which logic we refer to.) As known, the direct product $\mathscr{U}=\mathscr{T} \times \mathscr{S}$ admits exactly one state (see [6]); moreover, this only state attains 1 at all elements greater than or equal to the element $u=\left(1_{\mathscr{T}}, 0_{\mathscr{S}}\right)$.

Let $M$ be a countable set. For each $C \subseteq M$, let us take a copy, $\mathscr{U}_{C}$, of the logic $\mathscr{U}$. Let us now denote by $u_{C}$ the element of $\mathscr{U}_{C}$ corresponding to $u \in \mathscr{U}$ and let us construct the horizontal sum, $\mathscr{V}$, of the collection $\left\{\mathscr{U}_{C}: C \subseteq M\right\}$ (see [5] for the definition of the horizontal sum). Finally, let us construct our counterexample, $\mathscr{L}$, as a sublogic of the product $\mathscr{W}=\prod_{m \in M} \mathscr{V}$. We define $\mathscr{L}$ as the collection of all $f \in \mathscr{W}$ satisfying the following condition:

whenever $f(m) \in \mathscr{U}_{C} \backslash\{0,1\}$ for some $m \in M$ and $C \subseteq M$, then $m \in C$ and $f$ is constant on $C$.

First, let us prove that $\mathscr{L}$ is a logic. Trivially, $\mathscr{L}$ is closed under the formation of orthocomplements in $\mathscr{W}$. It remains to be shown that $\mathscr{L}$ is closed under the formation of countable orthogonal joins in $\mathscr{W}$. Let $\left\{f_{n}\right\}_{n \in N}$ be a sequence of mutually orthogonal elements of $\mathscr{L}$ and let $f$ be its join in $\mathscr{W}$. Suppose that $f(m) \in \mathscr{U}_{C} \backslash\{0,1\}$ for some $m \in M$ and $C \subseteq M$. All elements $f_{n}(m), n \in N$, satisfy $f_{n}(m) \leq_{\mathscr{V}} f(m)$, so they have to belong to $\mathscr{U}_{C}$. We see therefore that $m \in C$. All $f_{n}$ with $f_{n}(m) \neq 0$ must be constant on $C$. There is some $f_{i}$ satisfying $f_{i}(m) \neq 0$. For all $f_{j}$ such that $f_{j}(m)=0$, the relation $f_{i} \perp f_{j}$ implies that $f_{j}$ attains values from $\mathscr{U}_{C}$ on the whole $C$. Hence, $f_{j}$ is constant (equal to zero) on $C$. Thus, all $f_{n}$ as well as $f$ are constant on $C$ and, moreover, $f \in \mathscr{L}$. We have proved that $\mathscr{L}$ is a logic.

Notice also that $\mathscr{L}$ is a lattice (this was not required in [1] but it might be important in another context), though the lattice operations in $\mathscr{L}$ do not coincide with those of the lattice $\mathscr{W}$.

For each $C \subseteq M$ and $v \in \mathscr{U}_{C}$, let us denote by $v \mid C$ the element of $\mathscr{L}$ which attains the value $v$ on $C$ and which vanishes on $M \backslash C$.

Let us now define $\mathscr{P} \subseteq \mathscr{L}$ as the collection containing $0_{\mathscr{W}}$ and all elements of the form $\bigvee_{C \in \mathscr{F}} u_{C} \mid C$, where $\mathscr{F}$ is a finite collection of mutually disjoint subsets of $M$. Each element of $\mathscr{P}$ is of a finite length. One can check easily that $\mathscr{P}$ is closed under the formation of meets and that $\mathscr{P}$ is also finitelycoverable.

Finally, let us consider the state space of $\mathscr{L}$. Let $\omega$ be a state on $\mathscr{L}$. For each nonempty $C \subseteq M$, the interval logic $\{f \in \mathscr{L}: f \leq 1 \mid C\}$ contains a sublogic $\left\{v \mid C: v \in \mathscr{U}_{C}\right\}$ admitting exactly one state. We obtain that

$$
\begin{aligned}
& \omega(v \mid C)=\omega\left(u_{C} \mid C\right) \text { for } u_{C} \leq v, \\
& \omega(v \mid C)=0 \text { otherwise. }
\end{aligned}
$$

The logic $\mathscr{L}$ contains only elements of the form $\bigvee_{C \in \mathcal{I}} v_{c} \mid C$, where $\mathscr{I}$ is a sequence of mutually disjoint subsets of $M$ and $v_{C} \in \mathscr{U}_{C}$ for all $C \in \mathscr{I}$. Each state on the sublogic $\{1 \mid C: C \subseteq M\}$ of $\mathscr{L}$ extends uniquely to $\mathscr{L}$. Thus, all states on $\mathscr{L}$ are $\mathscr{P}$-regular but some of them are not $\sigma$-additive. The proof is complete.

\section{REFERENCES}

1. O. R. Béaver and T. A. Cook, States on quantum logics and their connection with a theorem of Alexandroff, Proc. Amer. Math. Soc. 67 (1977), 133-134. 
2. A. Dvurečenskij, T. Neubrunn, and S. Pulmannová, Regular states and countable additivity on quantum logics, Proc. Amer. Math. Soc. (to appear).

3. R. J. Greechie, Orthomodular lattices admitting no states, J. Combin. Theory 10 (1971), 119-132.

4. S. P. Gudder, Stochastic methods in quantum mechanics, North-Holland, New York, 1979.

5. G. Kalmbach, Orthomodular lattices, Academic Press, London, 1983.

6. P. Pták, Exotic logics, Colloq. Math. 54 (1987), 1-7.

Department of Mathematics, Faculty of Electrical Engineering, Technical University of Prague, 16627 Prague 6, Czechoslovakia 